\title{
Grandparents and Grandchildren Meeting Online: The Role of Material Things in Remote Settings
}

\author{
Verena Fuchsberger \\ verena.fuchsberger@sbg.ac.at \\ University of Salzburg \\ Salzburg, Austria \\ Philippe Bentegeac \\ philippe.bentegeac@sbg.ac.at \\ University of Salzburg \\ Salzburg, Austria
}

\author{
Janne Mascha Beuthel \\ jannemascha.beuthel@sbg.ac.at \\ University of Salzburg \\ Salzburg, Austria \\ Manfred Tscheligi \\ manfred.tscheligi@sbg.ac.at \\ University of Salzburg \& \\ AIT Austrian Institute of Technology GmbH \\ Salzburg \& Vienna, Austria
}

\begin{abstract}
Grandparents and grandchildren, who cannot meet face-to-face (e.g., due to dislocation or physical distancing induced by a pandemic), often use audio-visual communication tools in order to maintain their relationship online. In a qualitative online survey $(\mathrm{n}=85)$, we inquired into the various ways that grandparents and grandchildren came up with when being physically distant; many of them are tangible in nature as they include "things" or incorporate "spaces". In this paper, we illustrate related temporal and spatial trajectories and unpack how online meetings are characterized by constant negotiations of agency. We discuss how online meetings could complement face-to-face meetings, instead of mimicking or replacing them. We finally articulate a collection of design sensitivities with the aim to both inspire and question designing for intergenerational online meetings.
\end{abstract}

\section{CCS CONCEPTS}

- Human-centered computing $\rightarrow$ Empirical studies in $\mathrm{HCI}$.

\section{KEYWORDS}

intergenerational relationships, online meetings, audio-visual communication, materials, materiality, objects, spaces

ACM Reference Format:

Verena Fuchsberger, Janne Mascha Beuthel, Philippe Bentegeac, and Manfred Tscheligi. 2021. Grandparents and Grandchildren Meeting Online: The Role of Material Things in Remote Settings. In CHI Conference on Human Factors in Computing Systems (CHI '21), May 8-13, 2021, Yokohama, Japan. ACM, New York, NY, USA, 14 pages. https://doi.org/10.1145/3411764.3445191

\section{INTRODUCTION}

Maintaining relationships over distance and with the help of technologies has been of interest to the HCI and the Interaction Design

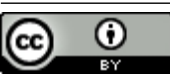

This work is licensed under a Creative Commons Attribution International 4.0 License.

CHI '21, May 8-13, 2021, Yokohama, Japan

(C) 2021 Copyright held by the owner/author(s)

ACM ISBN 978-1-4503-8096-6/21/05.

https://doi.org/10.1145/3411764.3445191 community for a long time. Questions of technological features and functions, material conditions and contexts have been widely addressed, and individuals' experiences and emotions have gained research's attention. For instance, research and design explored the needs for, and possibilities to, increase the feeling of presence (e.g., social presence or co-presence [8]). A variety of design concepts, prototypes, services, and products have been launched in the past years that individuals can make use of in distant relationships, such as between collaborators [46, 65], friends [10, 41], couples $[42,44,53,66]$, or families [3, 32]. The latter includes relationships between grandparents and grandchildren, for whom keeping in touch is often particularly important (e.g., [15]).

We, a group of $\mathrm{HCI}$ researchers, have spent the past few years exploring remote interactions for grandparents and grandchildren by means of tangible interactions in order to complement available tools that mainly allow audio-visual communication. At the beginning of our research endeavour, remote intergenerational relationships were often a consequence of living in different countries or cities due to international marriages or work relocations, but when Covid-19 quite abruptly required physical distancing, families living in the same street suddenly became dislocated; for many, meeting in person was not an option any more. This specifically affected relationships between grandparents and grandchildren, since due to grandparents' increased age it was recommended to be particularly careful and not meet. In response, an increase in audio-visual communication was widely observable (e.g., [64]).

Through conversations with grandparents and grandchildren, and also during our personal encounters with family members, we noticed that physical things seemed to be fairly important in such online meetings. Though this, in general, resonates with related work (e.g., $[18,60]$ ), we heard of and experienced numerous unexpected, innovative, and creative ways of how objects were included into audio-visual communication. In order to explore this observation further, we addressed this in our research by asking what roles physical objects actually play(ed) in the communication between grandchildren and grandparents during physical distancing. Our aim was to inquire into humans', but even more so, in non-humans' roles and their agencies, and how they are negotiated online. We decided to approach this objective by means of a qualitative online questionnaire, adapting to the situation also methodologically. 
The questionnaire was distributed in Austria in June 2020 and invited grandparents and grandchildren to share their experiences when meeting online during physical distancing with us in form of open-ended questions. In this paper, we summarize the accounts that 85 grandparents and grandchildren (68 from the perspective of grandparents, 17 from the perspective of grandchildren) described. We based our analysis on notions and vocabularies from ActorNetwork Theory, as this allowed us to foreground actors, activities, and agencies. We particularly looked into agency as it is distributed among diverse human and non-human actors in intergenerational online meetings, and what role they have, whether they are mediators (briefly said, they have an affect on the situation), or intermediaries (not having such any affect).

Based on this analysis, we contribute a discussion of approaches to remote online interactions between grandparents and grandchildren as we unpack (1) temporal trajectories, i.e., translations of offline activities and meaning to online activities and back; (2) spatial transitions, i.e., bringing the conversation to an actor in contrast to bringing the actor to a conversation; (3) effects and agencies of human- and non-human actors; (4) instabilities and dynamics of online interactions; and (5) "other" meetings, i.e., online meetings not replacing offline meetings, but considering (and eventually designing) them as something else. We finally articulate a collection of design sensitivities and implications that aim to draw attention to the particularities of the roles of material things in grandparents' and grandchildren's remote meetings.

We start this paper with an overview of related work in $\mathrm{HCI}$ in regards to intergenerational online relationships. We then depict the notions of Actor-Network Theory that we draw upon. Afterwards, we detail the method, describe the findings of the study, and follow with a discussion that results in five design sensitivities and according implications. We conclude by reflecting on limitations and potential future avenues for intergenerational online meetings.

\section{RELATED WORK}

Related work focuses on a variety of aspects of technology-mediated intergenerational relationships. It addresses, among others, technologies and tools that are used (e.g., $[9,51]$ ), activities that are conducted together (e.g., [25]), how smart toys are designed and adopted (e.g., [55]), how mentoring and learning works out (e.g., [68]), or how awareness for each other can be created in an ambient way (e.g., [7, 61]). In the following, we briefly summarize issues of shared spaces before we address particular strands of activities that are characteristic to grandparents-grandchildren relationships: playing, and reading and storytelling.

\subsection{Intergenerational Shared Spaces}

Many dislocated activities between grandparents and grandchildren are inspired by a co-located setting, where they share a common physical environment (e.g., reading books together [48], drawing together [25], or playing together [55]). Usually, these collaborative activities require a shared space, which is, if transferred to an online setup, constrained by the available technology, making "interaction through video-chats or phone-calls insufficient or even disappointing" [56, p. 62]. Even though researchers pay attention to the various requirements of video chats among distributed families (e.g., [26]), the absence of shared spaces can be problematic in intergenerational remote activities, since it may be be difficult for younger children to engage in focused interaction without sharing a sensory environment [56]. Thus, incorporating physicality and tangible objects seems to be particularly important for remote meetings as well, which we aim to investigate further in our work. This is not only relevant for children, but also for the older adults; for them, "material social communications afford expression of thoughtfulness and concern" [29, p. 3910].

In recent years there have been many attempts in the HCI community to develop systems that allow to create shared online spaces for intergenerational activities. For instance, "FamilySong" [56] is a system for grandparents and grandchildren to share, listen, and discover music together while being physically separated. Forghani et al. [21] suggested "G2G", a shared calendar and video messaging system that provides "grandparents and grandchildren with an awareness of each other's lives to support conversations and design elements to help reduce the need for parent scaffolding." [21, p. 1]. Some of the proposed technologies aim to strengthen the relationship by not letting parents interfere and allowing direct communication $[21,56]$, since limiting parental scaffolding has shown to result in a strengthened relationship between grandchildren and their grandparents [21]. We will discuss later in the paper that parental scaffolding did not come to the surface in our study and we will also provide some speculations why it did not.

\subsection{Playing}

Dislocated intergenerational relationships are often maintained via playful activities (e.g., [14]). Playing can present benefits for older adults such as improving a sense of well-being [49] or having positive effects on health, such as games for post-stroke limb motor rehabilitation [62]. Playing among grandparents and grandchildren might also positively influence their social communication [12].

In regards to video games, many children are used to this type of entertainment, for example, through mobile phones, computers or consoles, while older adults may feel disconnected from this type of 'digital culture' and game play; thus, designing for both age groups can present major challenges [11]. There have been been attempts to make up for this issue, such as "The Space Journey Game" [11]. It aims to allow both grandparents and grandchildren to feel competent by showing their (different) knowledge and skills. The authors conclude that designers need to be careful when designing digital games to not "make grandparents feel that they do not have enough skills to play with their grandchildren" [11, p. 6].

While playing is an important activity for grandchildren and their grandparents to stay in touch, findings show that not particular games, but playfulness is most important for intergenerational communication [14]. It is intrinsic to many intergenerational activities, involving "a range of activities including teasing, story-telling, the exchanging of significant objects, as well as games" [14, p. 209]. Consequently, it is to be expected that playfulness will be fairly important in dislocated intergenerational meetings; the way that it is enacted by means of objects is of interest in our study.

Starting from the idea that audio-video tools (though being better than audio-only) would not be appropriate for young children, 
as they were not specifically designed for family communication [18], Follmer et al. created a set of designs to explore alternative opportunities "for silliness and open-ended play between adults and young children" [18, p. 49]. One of their conclusions is that physical objects have the capability to spur discussion and interaction, which can also be real world objects such as the clothing that the children's mother would be wearing. They state that

Traditional digital interfaces can be very rigid and deterministic, but if we create them with an eye for improvisation children will be more free to explore and open to imaginative play. Tangible objects do not have to directly be sensed by the interface for them to scaffold physical interactions for children; our designs suggest they can succeed as props and topics for discussion. [18, p. 56]

We build on these findings and are particularly interested in whether or not the tools for audio-video communication are still considered insufficient (10 years after Follmer et al.'s work), how they are made use of, and what role objects may play today.

\subsection{Reading and Storytelling}

Further common interactions between grandchildren and grandparents are related to storytelling and reading. There is a growing interest of HCI in (e-)book reading within the family, with explorations of parent-child interactions while reading (e.g., [34, 40, 50, 59]), or grandparents and grandchildren reading together (e.g., [48]). For instance, there is evidence that when grandparents and their grandchildren read an e-book together remotely in their long-distance relationship, they have remarkably longer sessions in comparison to having mere video chats [48].

Research also investigated practices of telling family stories and sharing family history (e.g., [31, 37, 60]). Interviews with families revealed that stories are the result of co-constructive practices of both storytellers and listeners "proactively working to discover stories from different sources, deciphering stories to make sense of the content and meaning, and reconstructing stories" [31, p. 1].

The app "PicMemory" [37] aims specifically at improving the exchange and collection of family history. It allows family members to record their voice over pictures to share anecdotes related to the pictures and to collaboratively edit, rewrite, or summarize recordings. The app then regroups related pictures or stories together and thereby creates a collection of memories [37]. However, research also showed that grandparents seem to be more often the ones sharing stories or family memories compared to children [60], which might result in an unbalanced collection of memories. Given the various findings reported in related work that emphasize activities of reading and storytelling, we are curious whether physical objects support such practices, and if so, how they facilitate them.

We acknowledge that in $\mathrm{HCI}$ (and related) research a variety of ideas, prototypes, and systems have been proposed that already incorporate tangible interactions and which aim to strengthen the relationship between grandparents and grandchildren. Nonetheless, we start from the assumption that most grandparents and grandchildren will rely on audio(-visual) communication for their contact during physical distancing, since, to our knowledge, hardly any tangible interactions are available in current homes. We, hence, focus our studies on the means of communication that grandparents and grandchildren are most likely to use, and aim to understand how they make use of them to maintain their relationship over distance. In regards to feeling closer over distance, Lenz et al. concluded that "We aren't lacking technical possibilities, but knowledge of how to use this technology innovatively and successfully in everyday life" [38, p. 499]. We hypothesize that if there is no possibility to meet in person, such as during pandemics (or due to any other reason), users themselves will come up with innovative ways of using existing technologies, extending it, or altering it, to foster the relationship with loved ones. We start our work from this premise.

\section{BACKGROUND}

Epistemologically, the perspective taken in our work relates to the "non-human turn" [27], which emphasizes "that the human has always coevolved, coexisted, or collaborated with the nonhuman and that the human is characterized precisely by this indistinction from the nonhuman" [27, p. ix-x]. Coined as Entanglement HCI, Frauenberger suggests a similar perspective for contemporary $\mathrm{HCI}$ by engaging with entanglement theories. He suggests to "design meaningful relations, rather than optimising user experiences" [22, p. 2:22], an aspiration we share in our work.

In order to address both humans and non-human actors in the online relationships between grandparents and grandchildren alike, we turned to Actor-Network Theory (ANT) for guidance. ANT is not a theory, but rather an "open repository. A list of terms. A set of sensitivities." [39, p. 253]. At the same time, ANT provides "an analytical approach that examines how human and non-human entities co-construct dynamic and influential networks of associations, and the effects produced as a result of these networks" [23, p. 1]. This means that it allows to focus on the relations between different actors and how they - together - shape a situation.

ANT provides a great variety of concepts and vocabulary (e.g., [1]) that can be used as lenses to investigate social phenomena. We decided to review our work through the following four core concepts of ANT as they particularly align with our goal to account for human and non-human actors and their interrelation in remote settings (and which we will refer to in order to parse the data in our subsequently described approach):

- actors, i.e., someone or something that acts, does something, makes a difference [39], being either human or non-human (including objects, animals, tools, texts, etc. [52]); "everyone and everything can be an actor as soon as he/she/it evokes an action of someone or something else" [24, p. 2857].

- (relational) agency, i.e., the outcomes from performative relations between actors; this means that "material agency is uncovered and actors in the network can be followed to learn from them, to understand their location, their spatiality and therefore understand how each makes a difference in the network." [28, p. 16]; in other words, agency emerges between (human and/or non-human) actors if they have an effect in a given situation;

- intermediaries, i.e., entities that transport meaning or force without transforming it [36]; intermediaries do not have an effect on the situation; 
- mediators, i.e., entities that transform, translate, distort, or modify "meaning or the elements they are supposed to carry" [36, p. 39]; mediators develop agency and have an effect in a given situation.

Consequently, ANT provides us with the terminology and notions needed to inquire into objects and spaces, while retaining a focus on human actors. Taking this perspective on grandparentsgrandchildren online relationships will help us identifying actors, agencies, and the resulting transformations of unique situations through inquiring into individuals' heterogeneous approaches.

\section{METHOD}

We conducted a qualitative online study to explore the roles of physical objects in the communication between grandchildren and their grandparents during physical distancing. The notions of nonhuman-human networks and meaningful relations between them, as suggested in ANT, led us to design the questionnaire and analyze the collected data in specific ways. In regards to questionnaire design (which we detail below), we aimed to ask about how people would incorporate objects into their communication. We did not aim for quantitatively generalizable results, but for descriptions and narrations that would allow us to understand what is meaningful to grandparents and grandchildren (i.e., the human and non-human actors), what agency they develop in concert with the objects and their relatives, and how they react to the circumstances by means of technology. Furthermore, we aimed to distinguish between intermediaries and mediators in the actor-networks.

\subsection{Research Context}

This research interest is embedded in the research project re:tangent - remote tangible engagements ${ }^{1}$, a 3 -year research project, within which HCI researchers are conducting material explorations and user studies alike to explore physical / tangible ways of connecting grandparents and grandchildren over distance. Besides our aim to complement digital means of communication with tangible interactions, we also employ a critical approach towards designing for remote relationships. So far, this approach resulted in critical designs that manifest melancholic personal experiences of remoteness in a wearable (and as such, tangible) form [6] alongside designs to experience closeness together. With the study described in this paper, we aim to intensify the conversation about the various roles of $\mathrm{HCI}$ and Interaction Design in facilitating remote intergenerational relationships: Besides envisioning and designing novel technologies, we aim to draw attention to the importance of making visible, learning from, challenging, or supporting the numerous appropriated and situated practices that are already in place.

\subsection{Study Design}

We decided for an online questionnaire to be able to reach and include individuals, who were physically distancing due to Covid19. We were intensively discussing whether or not to approach individuals in these difficult times for research purposes. However, in various informal conversations (with colleagues, friends and family, who are grandparents or grandchildren themselves)

\footnotetext{
${ }^{1}$ http://hci.sbg.ac.at/retangent
}

we got the impression that people wanted to share their individual experiences and were eagerly doing so. We undertook various considerations and a pre-test (see below) to make sure the questionnaire was as sensible as possible to the situation. For instance, to avoid overwhelming participants with too many questions, we made the questionnaire as brief as possible (accepting the fact that we could not include all potentially relevant questions, such as how meetings looked like before the crises); we did not make questions mandatory to continue, and we did not collect any personal data beyond age and gender. We designed the questionnaire to not require too much time and effort, to not ask any dispensable questions and we emphasized that individuals can take as much or few time as they wish to answer our questions.

We designed the questionnaire to be qualitative in nature. Besides a few descriptive measures (e.g., to distinguish between grandparents and grandchildren and different constellations of respondents), we aimed for narrated experiences, meanings, and sensibilities through open questions. With this, we hoped to inspire respondents to reflect on their relationships and allow them to share individual stories rather than aiming for general, abstract, and, eventually averaged data. Since we target our study towards design sensibilities, we considered such qualitative data to fit our research goal best.

\subsection{Assessment}

Our research question (What roles do / did physical objects actually play in the communication between grandchildren and their grandparents during physical distancing?) can be disassembled into three parts, which were informing the definition and articulation of questions: the (audio-video) communication between grandparents and grandchildren, the role of physical objects, and pathways of contact during physical distancing.

After soliciting an informed consent (see below) and asking some demographic information (gender, age, and who would fill in the data, such as one or more grandparents, one or more grandchild(ren) with or without parents), we provided them with three blocks of questions (originally in German):

(1) Communication over distance: Do you use audio-video communication (e.g., Skype, Signal, WhatsApp, or Facetime) over distance in order to be in contact with your grandchild(ren) / grandparent(s)? If yes, what do you usually do when you are video chatting (with pre-defined categories such as talking, showing things, playing, singing, other)? What are you talking about, showing, playing, singing, etc.; if no, how had you been in contact when physical meetings were impossible?

(2) Objects over distance: Do you sometimes include "objects" into this communication, such as a plush animal, a brick, food, a doll, a flower, a desk, clothing, a board game, a book, or similar? If so, would you want to briefly narrate how that usually proceeds? Is there a specific reason for including this/these particular object(s)?

(3) Changes of being in contact over distance: How did the contact change over the course of the past weeks? What technologies would you like to see in the future to better support you in being in contact with your grandchild(ren) / 
grandparent(s)? What do you think will technologies never be able to accomplish?

While question block 1 and 2 were phrased in a straightforward way to assess the respondents' practices and experiences, block 3 inquired into the evolvement of practices. The reason for asking about wishes for technology and assumed boundaries was twofold. On one hand, we hoped to learn about experiences that they would want to make but did not, and experiences they were not even expecting. On the other hand, we aimed to provide the respondents with the possibility to express their overall attitude towards technology, and at the same time, motivate them to be reflective and critical. All questions were phrased in a first version based on the research interest, then intensively discussed within the project team and iterated several times before being pre-tested with six individuals (a convenience sample of two grandchildren and four grandparents). Pre-test respondents filled in the survey and discussed with us how they interpreted the questions and what they would do differently. Based on this feedback, we refined the questionnaire accordingly.

\subsection{Procedures}

The questionnaire was executed via LimeSurvey that is GDPRcompliantly hosted by the authors' university. We recruited participants via local networks (e.g., senior citizen communities, knowledge institutions) and via our university's and institution's public channels (e.g., news feeds, social media). The questionnaire was available for 4 weeks in June 2020 (when physical distancing was still recommended in Austria, but not enforced any more). We invited grandparents and grandchildren (without any restrictions) to fill it in once; no compensation or incentive was provided.

In the beginning of the questionnaire, participants were presented with study details (goals, procedure, data collection, time needed, etc.), the research project it is part of, and contact details. We clarified that grandchildren below 18 years needed consent by their parents, and were invited to fill the questionnaire in together with their parents. Afterwards, details regarding data collection were displayed (an easy to understand summary of participants' rights; details why, what kind of, and how the data was collected). Finally, we asked them (or their legal guardians) for their consent. After filling in the questionnaire, respondents were dismissed by thanking them and again listing ways of getting in touch with us.

\subsection{Participants}

Overall, 123 individuals accessed the questionnaire, whereof 72 completed it. From the incomplete questionnaires, we included those into the analysis that comprised data beyond demographics, i.e., they all contained substantive and original qualitative data. This resulted in 85 questionnaires to be analysed. Thereof, 68 questionnaires were filled in by grandparent(s), and 17 by grandchild(ren). As mentioned earlier, we only collected participants' age and gen$\mathrm{der}^{2}$; even though both data were voluntary, all but one grandparent added them. Table 1 provides an overview of the participants.

\footnotetext{
${ }^{2}$ In order to assess gender, we considered the most feasible way that would take diverse gender identities into account, but that would also speak to grandparents' and grandchildren's conventionalities. Since in the area that the recruitment mainly took place the singular use of "grandparent" is not common, we decided to ask them whether they would be grandmother, grandfather, both together, or other. For grandchildren, it is common here to use the singular word "grandchild", hence, we provided them with
}

54 grandparents indicated to have used audio-video communication with their grandchildren, in contrast to 10 who have not (4 answers were missing). From the overall 17 grandchildren filling in the questionnaire, 11 indicated to have used audio-videocommunication with their grandparents (6 did not). Respondents who indicated to not use audio-video communication mentioned to make use of calls, WhatsApp, emails, letters and post-cards, texting, or to be in co-located contact (e.g., over the fence, on the balcony).

\section{RESULTS}

The quantitative data was analyzed descriptively (calculating means, standard deviations, and ranges, if applicable) and provides additional context (e.g., participants' age mean and range) to the core of the analysis, which consists of the qualitative data. We did deliberately not relate the quantitative data with qualitative findings due to the aim of the study and the respective sample size - it would be invalid to deduce any correlations, let alone causalities.

We also refrained from counting similar answers to result in frequencies (of activities, of objects, of conversation topics) due to the fact that they might be heavily blurred. For instance, we do not know whether a particular grandmother, who responded, would be linked to a particular grandfather, who responded as well, while others would fill in the information jointly. Consequently, any count would very likely not represent the situation appropriately.

The qualitative data, eventually, was analyzed by means of content analysis, which proceeded as follows: One author of this paper went through all data to check for unclear answers, then translated the answers into English and paraphrased them. Having been working with notions from ANT for several years, this researcher translated the notions from ANT into initial codes and coded the data accordingly: actors in online meetings between grandparents and grandchildren were spotted; remote activities were analyzed towards their agency and how it is distributed among actors; mediators and intermediaries were identified.

The data was then analyzed in terms of "transformations", i.e., practices that grandparents or grandchildren reported to have changed, in order to shed light on the meandering agency and how things became mediators in the relationship between grandparents and grandchildren, not only online but also offline. Finally, boundaries of online meetings were analyzed in order to understand what the grandparents and grandchildren experienced as lacking or insufficient in how they currently meet online. Then, the findings were condensed on basis of the discussion with all authors, thereby establishing an inter-subjective understanding.

An example: the transformative qualities of the ANT notion "mediators" were translated into three initial codes [existing object / space / activity before lockdown], [object / space / activity appearing or emerging during lockdown], [object / space / activity remaining after lockdown]; in the subsequent discussions among all authors, we agreed that if data was coded this way it characterized mediating actors and denoted a "transformation". In this interdisciplinary group, also the design sensitivities were deduced and articulated, which are presented in the discussion section 6 .

an open text field to indicate their gender without predetermined answer categories. In the analysis, we coded those answers accordingly. 
Table 1: Study Participants: demographics, including constellations of filling in the questionnaire, their age and gender. *Since respondents could fill in the questionnaire in groups, the numbers of data sets does not necessarily equal the sums of gender identity indications (while we have 17 data sets that represent grandchildren, we have 18 indications of gender identities).

\begin{tabular}{lrlr}
\hline Grandparents & $\mathbf{6 8}$ & Grandchildren & $\mathbf{1 7}$ \\
\hline Filled in by a grandmother & 32 & Filled in by a grandchild & 11 \\
Filled in by a grandfather & 31 & Filled in by a grandchild with parent & 5 \\
Filled in by grandmother and grandfather & 4 & Filled in by several grandchildren with parent & 1 \\
Other & 1 &
\end{tabular}

$\begin{array}{lr}\text { Grandchildren's gender } & \\ \text { Female } & 12 \\ \text { Male } & 6 \\ \text { Other } & 0\end{array}$

\begin{tabular}{|c|c|c|c|}
\hline \multicolumn{2}{|l|}{ Grandparents' age in years } & \multicolumn{2}{|c|}{ Grandchildren's age in years } \\
\hline Mean & 69.13 & Mean & 18.17 \\
\hline Standard Deviation & 7.25 & Standard Deviation & 10.63 \\
\hline Min & 54 & Min & 5 \\
\hline Max & 85 & Max & 44 \\
\hline
\end{tabular}

\subsection{Intergenerational Online Meetings}

The respondents reported a variety of activities that they would perform when meeting by means of a video chat:

- Talking to each other about objects, such as a book, a toy, food, digital homework; about places such as school, kindergarten, or university; about conditions, such as health, sleep quality, the weather, the TV, sorrows, or problems; about special events or incidents, such as particularly pleasant or current events; about something that just occurred to them in the situation; about the future, such as plans or their envisioned reunion; about achievements such as whether the grandchildren would have learned new words or improved their foreign language skills; about activities, such as daily routines and recreational activities, their hobbies, hiking, games they just had played; about social networks, including family, friends, and pets; about their emotional bonds, such as how they miss each other, reciprocal solace and encouragement; about world affairs, such as the news, current topics in politics and science; small talk, including trivialities, jokes, and life overall.

- Showing pets, such as the cat; spaces, such as the apartment, the garden; objects, such as new games, books, toys, drawings, pieces of handicraft, a self-made cake, a particular table decoration, the children's clothing, flowers, furniture, selfbuilt lego buildings, children's hand-written texts, things that they connect with each other; activities, such as grandchildren showing what they are currently doing, grandchildren singing or playing an instrument, searching for easter eggs in the garden; messages, such as a particular WhatsApp message, something funny, or photos; deliberately showing themselves to each other.

- Playing with Punch puppets, building bricks, board games, card games, ludo, or dice games.
- Other things, such as singing and dancing, pattering, walking through the house and visiting every room, learning together, reading out, joint family celebrations (including touching glasses), or making faces.

Many of these activities, including motivations and rationales, were described in detail by the respondents. They mentioned that they would show particular objects in order to demonstrate something, such as the children's clothing revealing something about the activity that they did (e.g., sports clothing telling about the kind of sports that the kids were doing), or when grandparents were shown around in the children's room as a demonstration of how the children had tidied up. Some respondents indicated to perform a particular secondary activity for the online meetings to work out, such as the cat being allured to come into sight by means of treats: "the house cat; usually does what she wants... often one only sees her bushy tail captured [by the camera], but can be lured by means of a treat; additionally, the cat's toy (a small plush rat) was treated with valerian, so that the cat feels like playing right there where she can be seen" [grandparent, 56y]. Furthermore, some mentioned a particular requirement for the meetings, such as both sides having the same books available in order to, for instance, take turns in reading out or discussing the book, or to do exercises together remotely.

Overall, a number of further individual practices were reported, including objects to engage each other, such as a stuffed animal that talks to the grandchild in order to engage it in a dialogue or that directs grandparents to lego buildings and guitar exercises, or distributed physical setups, such as having a board game at one location and the die at both (e.g., grandparents throw a physical die at their home, a grandchild moves the grandparents' physical token at the board game that is set up at the grandchild's location, with self-made name tags added to the board game for all co- and dislocated players). Furthermore, space explorations were mentioned, when the grandparents showed the child around in the child's room at the grandparents' house, when the child was 
shown around in the grandparents' workshop to unveil what the grandfather was working, or when craftspeople's activities at the grandparents' house were watched together. Other playful actions, such as the grandchildren sending emojis after the grandparents had read out to them, or the grandfather making faces to make the grandchild laugh were also described.

Hence, numerous human and non-human actors were involved in the online meetings between grandparents and grandchildren. In terms of human actors, besides the grandparents and grandchildren themselves, also parents and friends or craftspeople became direct or indirect part of the meetings. Several non-human actors seem to play a role, which differ in their attributes. Among others, they can be living (e.g., pets, plants), inanimate (e.g., easter eggs, dice, photos), material (e.g., stuffed animals, guitars, clothing), immaterial (e.g., sleep quality, a family celebration, sorrows), and/or digital (e.g., digital exercise sheets).

\subsection{Role of Objects}

As detailed in section 3, we analyzed the data in regards to their effects on other actors, i.e., whether or not they would convert meaning. We identified several intermediaries (entities that transport meaning without having an effect on the network), and a considerable number of mediators (entities that do have an effect). Before we list these intermediaries and mediators, we illustrate their distinction by prepending one example. When the camera that grandparents and grandchildren use for remote playing is adjusted to the game, it is not itself affecting the situation (i.e., it is an intermediary), but is affected by it. In contrast, the game affects the camera as it requires the camera to be repositioned by the grandparents, it reveals agency, and thus, qualifies as a mediator.

5.2.1 Intermediaries. Similar to the camera that does not transform meaning, but transport it, easter eggs, bushes, flowers, or table decorations were mentioned to be shown in online meetings while not revealing any transformative power. All those actors that are involved into online meetings to demonstrate something may be considered to be intermediaries, such as a cake demonstrating successful baking to each other, drawing demonstrating pride, the garden demonstrating the growing of plants, self-made things demonstrating what has been tinkered with, things demonstrating what the easter bunny got the grandchildren, surroundings demonstrating how someone is living, etc. Similarly, actors that have been reported to initiate conversations, also do not necessarily transform meaning, such as food shown, the weather, the TV, pictures, or toys. Some other actors were mentioned to be used as part of an activity, such as a book being read out, or music being played and a video thereof being sent to the grandparents. Furthermore, audio-video communication hard- and software does not necessarily affect the other actors. However, it can do so, as described in the following, and act as a mediator.

5.2.2 Mediators. Respondents described how they brought objects to the meeting. For instance, they would position a metal bird in front of the camera, wind it up and have it run to entertain the grandchild. Alternatively, the technology was reported to be carried around to show each other around in the apartment, workshop or garden: "the children's room, the garden and how everything is growing" [grandchildren with parent, 9y and 11y] or "if possible, we adapt to the grandson's wishes: he would like to see the room, where he, when he stays with us, sleeps. Or he would like to see the cellar, where the grandfather is currently working" [grandparent, 68y].

The physical properties of the technology, hence, enabled different usages which had an effect on how the online meeting proceeded. Furthermore, respondents described that the technology would provide them with a less real impression of daily routines, with developmental steps of grandchildren becoming less visible to them. Additionally, limitations of the technology may substantially influence, even distort, the online meeting: respondents reported issues with bandwidth, lacking quality of webcams, etc.

Besides the technology itself being a mediator, respondents described many accounts of when other - non-human - actors influenced the online meetings. For instance, the just mentioned wind up toy influenced how the online meeting proceeded. The respondent reported that it gained the child's attention, and thus, it enabled being together for some time. Similarly, the stuffed turtle was eventually engaging the child, not the grandparent, or punch puppets were conveying a mood themselves. Toys were revealing what the children played, pictures were accentuating narrations, jokes were cheering up the children, or cookies resulted in the child wanting to grab them (who grabbed the phone instead). Even more, the mediators were reported to have effects on the frequency, duration, and emotional experiences of online meetings. Respondents mentioned that learning together, which was only enabled by the shared digital homework or the existence of two identical physical books, would result in meeting daily, in a warm atmosphere. Playing remotely together, reading, and learning would result in more frequent contact, "Eventually I saw my grandchildren, even if only virtually, much more often than usual" [grandparent, 76y].

\subsection{Transformations}

Looking for agency (i.e., transformative outcomes of the performative relations between actors, see chapter 3) in the reported accounts, we found a variety of transformations that the respondents described. They became visible when respondents reported activities and practices that had roots in their previous physical meetings, when they described what they developed during online meetings, and what they carried forward to their face-to-face meetings after physical distancing ended. There were reports about "things" that were meaningful for the respondents before they started to meet online, such as a stuffed toy they had played with together when meeting face-to-face, that was forgotten at the grandparents' place and that, during the online meetings, conversed with the child online as well. Games were mentioned that they had played together before and continued to play in online meetings, or cats that had been with the grandparents, loved by the grandchildren and involved in the online meetings, etc.

Some practices were reported to have emerged during, or were enabled by, the online meetings, such as a particular toy that appeared for the first time in an online meeting, or the possibility to watch each other's face for a long time, which would be awkward in a face-to-face meeting: "In normal contact you can never look into the other face for such a long time. Every time my heart was melting when seeing these beautiful and lovely children" [grandparent, 
76y]. Further examples related to learning in an individual manner (e.g., developing dislocated learning routines with three grandchildren separately and differently), or to rules that evolved based on a dislocated game setup. Daily routines were being established for reading out: "It felt as if we would visit them every day" [grandparent, 68y]. Emerging feelings were also reported, both negative and positive ones, such as alienation, or the pleasant anticipation of meeting again soon. It was also mentioned that changes were not only induced by the necessity of physical distancing, but by the individuals' situation. For instance, a grandparent explained that the contact did not change tremendously due to the physical distance, but "he usually lives with me for two months a year, when he is in town for vocational school. At the moment, he is in love and his girlfriend is more important than his grandmother, but that's ok for me" [grandparent, 68y].

Since the questionnaire still ran when the physical distancing measures were loosened, some respondents also described how the previous online meetings influenced their subsequent face-to-face meetings. Due to frequent online meetings, several respondents indicated that the relationship appeared to be even closer than before. Eventual face-to-face meetings were experienced very intensively and consciously, feeling, for example, immensely grateful to be able to meet in person again. Others reported that online meetings continued even when meeting in person was possible again, not to replace the face-to-face meetings, but to complement them. Some objects that were (or became) meaningful to them in the online meetings were mentioned to endure, such as toys playing a distinct role in the eventual face-to-face meetings as well. "I wanted that [the grandchild] gets a positive feeling by remembering details from her visits with us and that she can link the visits with this feeling. And that [she] does not lose memory of us. This worked out, during her first visit [after the physical distancing] she got the [toy] cat out of the cupboard and wanted to go to the pantry, where I always keep the cookies...." [grandparent, 59y]; the cookies that had been shown during the online meetings.

\subsection{Boundaries of Online Meetings}

While in general the respondents seemed to be satisfied with how the technology served them in meeting online (e.g., they indicated to be pleased / happy with what is available, finding the technology sufficient / great, with wonderful / endless possibilities), several boundaries of online meetings were also mentioned. They most prominently referred to the bodily, emotional, and experiential aspects of relationships that online meetings can not sufficiently cater for. Bodily closeness was mentioned to be lacking, including the visible, sensible, warm, and palpable body, or the possibility to hug, touch, kiss, and hold, resulting in an incomplete feeling of the other one; what is lacking are "hugs, the feeling of a human "aura". Even if today there are already diverse [VR] glasses, where such attempts are being made" [grandparent, 74y]. Emotional closeness, including caress and rewarding, was also considered insufficient, or the experience of physical presence, since such meetings would not "be real", even though they would be transmitting "real" feelings via mimics and voice. There were reports that the additional medium would result in even more distance (though being "better than nothing"), or that they would aim to replace face-to-face meetings but fail in doing so, since, for instance, the whole environment would be missing: "I cannot estimate how the technology will change, but it will definitely remain impersonal" [grandparent, 78y]. The physically present sensory richness was mentioned to be lacking, with the consequence that not all senses would be sufficiently addressed (e.g., smell). A grandparent indicated to wish for "the transition of smell... Children often also connect a particular smell with their grandparents. (Deodorant, for instance, also black tea with a dash of rum, that sort of thing)" [grandparent, 62y]. Finally, being human was questioned to be possible online, it would rather be the medium that regulates (i.e., mediates) the relationship.

Some respondents also mentioned lacking opportunities compared to physical meetings (even if they would be existing): "I undertake many things outside the home - you cannot go into the woods or to the museum online and experience something together" [grandparent, 78y]. Not just since the Covid-19 pandemic, but increasingly since then, many virtual activities would have been available (e.g., virtual museum visits at the Museum of Modern Arts in New York ${ }^{3}$, or the Musée d'Orsay in Paris ${ }^{4}$ ) for grandparents and grandchildren to be incorporated into their online meetings, but they were not.

Further responses referred to technical, organizational, and security limits, such as the technology not being wanted every time and everywhere, the danger of being eavesdropped, questionable data protection, or too many advertisements. Furthermore, complex, erroneous, and expensive technology and Internet connections were mentioned as boundaries for grandparents (not only by themselves, but also by the grandchildren expressing the requirement for easy-to-use, intuitive, or simple interfaces for their grandparents); "Everything should be designed a little more simply - some things are really complex" [grandchild, 22y].

Overall, the study unveiled manifold activities that grandparents and grandchildren conducted together online, showed various roles and effects of human, and in particular, non-human actors, and provided insights into transformations that (online) meetings went through. Additionally, missing (sensory) qualities of such meetings became visible that limit the perceived opportunities for meeting online. The subsequent chapter discusses these findings and interprets them in regards to HCI research and design.

\section{DISCUSSION AND DESIGN SENSITIVITIES}

In the following, we discuss the findings and relate them to scientific work. In order to articulate the conclusions from this discussion, we depict five design sensitivities and resulting implications that aim to inspire and provoke (design and $\mathrm{HCI}$ ) research and interaction design(s). We do not propose one particular way of designing (for) intergenerational online meetings, or one particular design, but suggest directions that seem to be promising to research further, to consider in design(s), or to question and discuss.

\subsection{Temporal Trajectories}

As illustrated earlier, there is a variance in (kinds of) objects that grandparents and grandchildren incorporated in their online meetings. The respondents of the survey explicitly mentioned several

\footnotetext{
${ }^{3}$ https://www.moma.org/magazine/articles/267

${ }^{4} \mathrm{https} / /$ artsandculture.google.com/exhibit/from-station-to-the-renovated-musée-dorsay/ARK7SK5T?hl=en
} 
objects, and further human and non-human actors were part of the descriptions that they provided. Besides the grandparents and grandchildren themselves being human actors in the reported networks, including the role of their bodies and resulting possibilities for activities (e.g., making faces), further human actors were identified to play a role, such as craftspeople to watch together. The children's parents, who have been reported in related work to often take a scaffolding role in the relationship between grandparents and grandchildren [21], were, however, not mentioned by our respondents. This might be due to the fact that parental involvement is often related to organizational or technical issues, such as helping children with means of communication or scheduling (e.g., $[5,20,21])$, which we did not ask about in our study.

In regards to non-human actors, the findings showed that (living or inanimate, material or immaterial) objects functioned as conversation starters, they visualized everyday activities, facilitated shared activities, or served as reminders of each other or of events, as memento, or as investment into the future (relationship). Forghani and Neustaedter already pointed to the importance of reminiscing about mutual memories in cases of recent separation [19], which seemed to be the case for several of our respondents as well. Ames et al. described a benefit of video chats for children in that they can place objects (or bodies) "in front of the camera rather than needing to find words to share" [2, p. 147], a benefit that we also identified in our study. In contrast, we did not find that children would use objects to block the camera, as described by Ames et al. [2], but identified a huge range of activities that are supported, or even only enabled, by objects.

The importance of such non-human actors is also evidenced through their temporal trajectories. Non-human actors seem to have a bridging function between face-to-face meetings and online meetings. Additionally, we identified practices and characteristics that decreased during physical distancing, and some that increased; however, these were inherently individual, even intra-individual.

6.1.1 Design Sensitivity \#1. Objects seem to play a central role in intergenerational relationships, and have heterogenous trajectories: some become meaningful during physical meetings, are transferred to online meetings, and transferred to subsequent offline meetings; some become meaningful in online meetings with or without an effect on following offline meetings. Design may focus on how to enable objects to traverse online and offline rather design a particular object; it may rely on histories and memories as much as on the aim to create memorable experiences.

6.1.2 Implications. A number of designs have already been proposed that comply with this sensitivity. For instance, the wooden "StoryBox" allows grandparents and grandchildren to share photos, tangible artifacts, or audio recordings of everyday life over distance [60]. Their system builds on asynchronous sharing, and does not rely on a screen. In a field study they found that the "StoryBox" indeed served as an interface for sharing non-digital content, such as hand-made objects, photos, or food. All of these objects were also found in our study to be of relevance over distance, even if sharing was not particularly supported by the technology. In addition to allow sharing existing objects, it might, however, also be worth exploring objects that can transcend the physical through living a parallel digital life. As a starting point, we could imagine "quantified toys" whose conditions could be monitored or even enhanced by catering for both the physical object and the digital version, individually and together, synchronically and asynchronically.

\subsection{Spatial Trajectories}

While the just mentioned "StoryBox" seems promising to share tangible objects over distance, it is restricted in that it only allows a particular size of objects to be shared, at a fixed place. It also does not support grandparents and grandchildren showing each other around in and around their homes. The subsequent design sensitivity, number two, addresses this aspect:

6.2.1 Design Sensitivity \#2. Intergenerational online meetings seem to establish different spatial trajectories: they bring physical objects to the location of the online meeting (e.g., toys brought to the place that the smart phone sits at), or they are brought to specific places (e.g., the smart phone being dragged around in one's apartment or garden). Design may focus on dynamic spatial configurations rather than on proposing and relying on a specific one.

6.2.2 Implications. Reinterpreting what Dalsgaard et al. suggested for design research projects, namely designing for boundary zones [13], may also be worthwhile for intergenerational online meetings. Boundary zones are conceptual spaces where different stakeholders meet, and which involve shared artifacts and physical spaces that can "scaffold joint exploration and action" [13, p. 748]. Similarly, online meetings of grandparents and grandchildren may be considered boundary zones, which are constraining and enabling their shared experiences alike, both in temporal and spatial terms. One respondent expressed the idea to have a dedicated video-conferencing room with a big screen and a camera for the intergenerational meetings; however, it might be advisable to focus on spaces that are already significant in grandparents' and grandchildren's everyday life to allow for meaningful transitions and temporal trajectories across physical and digital spaces. This would call for explorations and designs of boundary zones that make use of digital possibilities (e.g., seeing each other over distance, creating accessible memories through recordings) and physical properties (e.g., furniture in specific rooms, flowers in the garden) at the same time, where meeting each other does not mean to disregard the individual physical surroundings, but explicitly include them during the online meeting. For instance, the concept of distributed hide and seek [58] could be revisited as it combines digital and physical spaces.

\subsection{Agencies}

Spatial trajectories of human, and in particular non-human, actors in intergenerational online meetings are also related to a distinction we focused on earlier in this paper, i.e., between intermediaries and mediators. As long as we consider the technology (e.g., the mobile phone, the laptop, an app) as an intermediary, and as such, not affecting the communication, the various dynamics that emerge may not come to the foreground. By explicitly asking whether they do have effects (e.g., not only allow, but afford mobility) on specific activities rather than considering them as "uninvolved" mediators, a 
variety of activities gets visible that seem to be important for grandparents and grandchildren when meeting online. This is reflected in the next design sensitivity:

6.3.1 Design Sensitivity \#3. Human and non-human actors can, but not necessarily have an effect on online meetings, i.e., they are intermediaries or mediators. Design may play with these differing roles and consider them explicitly; actors may meander between the different roles and thereby co-create novel experiences and unique situations.

6.3.2 Implications. The findings revealed numerous, orchestrated, small and subtle, actors mediating what is happening, contributing to the overall experience of the grandparent and the grandchild The responses also unveiled that many online meetings seem to be carefully considered and planned by including objects and technology in novel and thoughtful ways. In order to enable technology to meander between affecting the situation and being affected, we may reconsider the basic technology that is used in audio-video communication: What if a drone provides the video? What if we have the camera sitting on one's shoulder, just like a parrot? What if we don't see the other person on a separated computer screen (if at all), but projected on or next to a meaningful object? By doing so, the technology might either become less prominent, not requiring the meeting to be fit to the technology if desired, or become even more prominent (e.g., the parrot), being a playful non-human actor and thereby even be a participant in the meeting.

\section{4 (Re-)Negotiations}

The respondents did not report to have used any predefined or -designed online activity (as unveiled, for instance, by wishes to go to the museum together, without realizing that this would be possible online as well). We speculate that this is related to what we described earlier, i.e., that most of the respondents found the technology itself sufficient, maybe not even considering or searching for something more sophisticated, but relying on their own creativity and familiarity with each other. In 2010, Follmer and colleagues stated in regards to video-conferencing for long-distance families that "the tools themselves are not designed to accommodate children's or families' needs" [18, p. 49]. Though the tools certainly matured, they are still not designed particularly for their needs. However, based on our findings we postulate they do not even need to, since grandparents and grandchildren, in concert with a variety of non-human actors, seem to come up with their own ways of making use [57]. This results in the following design sensitivity:

6.4.1 Design Sensitivity \#4. Physical distancing resulted in a whole new set of yet-to-be explored practices and experiences, hence, established routines are fragile. Research may further inquire into individual approaches to online relationships and constantly question existing knowledge and, even more, predictions regarding what routines will endure. Design may not focus on a particular instability, but on the possibility that instabilities occur that reshuffle how people have organized their lives and developed their routines.

6.4.2 Implications. This is already substantially reflected in HCI, with "situated action" [54] being one of the most prominent notions, or "computer-supported practices" [35], as a younger, but increasingly influential one. Practices in online meetings may be individually established and constantly re-negotiated - with no set rules, practices, behaviors. Co-inhabiting a shared online space sometimes implicitly, sometimes explicitly, may involve the cocreation of a shared presence, the recollection of history, the cointerpretion of what is going on, how it feels, how it works out, and how a dislocated, yet shared physicality, might influence the future relationship. In order to design for such instabilities it might be promising to provide grandparents and grandchildren with the mechanisms to share physicality in a digital space, e.g., through exploring physical proxies in remote settings (e.g., [17]) or through enabling physical objects to relocate across distance (e.g., [43]), rather than designing dedicated online activities (e.g., [48]) that are restricted in how they can be transferred to physical meetings or changing circumstances.

\subsection{The "other" Meeting}

(Spontaneously) establishing new practices did not happen without frictions. Some of the reported frictions were related to technology and how well it worked for the heterogenous users. In our study, technical issues were rather ascribed to the grandparents, either by themselves or by their grandchildren ${ }^{5}$. This is similar to what Priego and Wilkins [47] also recently described; they reflected on their own experiences of videoconferencing with ageing relatives in form of comics, starting from initial discomfort experienced by the older participants and resulting in enjoyable meetings. They used comics "to visualize the idea that videoconferencing platforms can serve effectively as substitutes for physical presence - if not perfectly, at least closely enough to afford satisfaction to all participants." $[47$, n. p.]. The question of whether online meetings, however, can substitute for physical presence has been raised by our respondents. This discussion was mainly related to emotional shortcomings, maybe best summarized as the "aura" that would be missing. The mediated presence was not perceived as a "real" presence, and the lacking possibility to hug and touch each other was considered particularly problematic; skin hunger [45] was expressed.

Many respondents framed the boundaries of technology along the lines of replacement, emphasizing that offline meetings could (and should) not be replaced by online meetings if meeting faceto-face is an option. However, we also found accounts of when online meetings, which initially replaced the face-to-face meetings during physical distancing, were then held in addition to physical meetings, opening up a mixture of meeting experiences, frequencies, and activities. Taking these reservations and opportunities for novel practices as a basis, we articulate a final design sensitivity:

6.5.1 Design Sensitivity \#5. Online meetings often seem to be considered with reservations; they are lacking bodily qualities, they offer insufficient expressive qualities, and they may even be perceived as threats to physical meetings. Thus, research may question existing solutions or concepts and design may (critically) explore "other" forms of meetings, "other" activities, objects, places, and meanings. Novel experiences and values might be envisioned, rather than aiming to replace one kind of meeting by the other.

\footnotetext{
${ }^{5}$ This "second-hand user experiences" may be focused on in future work, aiming to investigate further how, for instance, younger people experience older ones' difficulties or even only pre-assume such, independent from their existence.
} 
6.5.2 Implications. Starting points for such considerations were already provided by respondents: one indicated that in online meetings, in contrast to offline meetings, one can observe the other one's face for a longer time, expressing an added value; another one described how grandchildren would send emojis after reading out from a co-existing physical book, pointing to a hybrid activity. Both examples would not be possible, nor make sense, in offline meetings. Furthermore, we might critically revisit technologies that mimic behaviors and practices in offline relationships (e.g., hugs over distance [42], remote breath signal sharing [33]), and instead explore designs of novel "other" spaces. These explorations may aim to manifest virtual wonderlands or magic worlds in order to allow designers (and users, if design is understood as a participatory endeavour) to detach from existing physical meeting practices and spaces, and to envision the "other" in an intergenerationally appropriate way.

\subsection{Outlook}

The design sensitivities and their resulting implications are attempts to create an awareness of what matters to grandparents and grandchildren, how they in combination with other human actors and heterogeneous non-human actors establish meaningfulness. At a minimum, grandparents and grandchildren require a medium to connect in order to create their own, unique setting. Complementary, a variety of sophisticated designs might support grandparents and grandchildren being together online, many of such have been proposed already for sharing existing physical objects (e.g., [60]) or perform a particular, object-dependent activity together, such as remote playing $[30,67]$ or eating [63]. Based on our findings, this list could be expanded by feeding a cat together, searching for easter eggs remotely, or sneaking into each other's rooms. However, it seems that grandparents and grandchildren do not just aim for digitally-mimicked activities, but for additional ways to engage that harvest the dislocated settings' potentials rather than jeopardize co-location. This calls for questioning existing solutions and critical designs, and bears great opportunities for novel hybrid, dislocated online meetings that we aim to explore further in our research.

\subsection{Methodological Reflections, Limitations, and Disclosures}

The analysis and the design sensitivities may have looked differently, if we had chosen another theoretical frame. We relied on ANT as it provides us with a view on agency and a vocabulary that favors competences over intentions. However, if investigated from, for instance, a socio-materiality point of view, we might have had findings towards material and immaterial aspects of organization (e.g., parental scaffolding) or collaboration (e.g., how actors negotiate goals and achievements), just to name a few.

Further, our study faces some limitations, e.g., due to the uneven number of grandparents and grandchildren participating, with grandparents being the majority, or the broad age range of grandchildren. These limitations require us to understand and interpret the findings as particular to grandparents, but not necessarily to (a non-homogenous group of) grandchildren, and to continue with investigations that include both perspectives equally, potentially together with the parents' perspective. Further, the findings are specific to the current Covid-19 pandemic and how grandparents and grandchildren reacted and adapted spontaneously. Interpreting them towards longer-term separations or other forms of dislocation (e.g., between parents and children, friends, or colleagues) is, thus, remaining on a level of speculations.

Overall, it is a sensible topic we are dealing with, one that affects us as researchers as well. We, the authors, are also experiencing the pandemic with all its disadvantages; we are parents; we are grandchildren. Our own experience of the situation (e.g., us observing our children video-chatting with their grandparents, we ourselves being in contact with our grandparents) certainly influences how we phrased the survey questions, how we analysed the data, and how we interpreted the findings. In order to avoid a systematic bias, we were continuously discussing our findings and interpretations with colleagues. It is, however, our own reading of the data that eventually defines what we describe, and how we describe it.

\section{CONCLUSION}

This work investigates pandemic-induced online meetings between grandparents and grandchildren. In a qualitative inquiry (online questionnaire; $n=85$ ), we identified a variety of human and nonhuman actors that shaped their experience during a period of not meeting face-to-face, including human actors such the grandparents and grandchildren themselves, or craftspeople they were watching together, as well as, non-human actors such as pets, cookies, table decorations, or music instruments. Our study revealed that such intergenerational online meetings seem to be creative encounters where new practices and activities are constantly being developed and enacted. We contribute five design sensitivities, which highlight that these online meetings may be characterized by

- temporal trajectories, manifested through objects or spaces that had been meaningful in the relationship between grandparents and grandchildren before physical distancing, became meaningful during online interactions, or lasted beyond online meetings;

- spatial trajectories when grandparents and grandchildren visit each other digitally in particular apartments, rooms, gardens;

- incorporated objects and spaces, whose agencies and effects are continuously re-negotiated;

- an instability as they constantly question the situation and reinvent what is happening; and finally,

- reservations in regards to whether or not they will be carried forward when meeting in person is possible, depending on whether they add to face-to-face meetings, not mimic, not replace, not substitute them.

While this study specifically referred to intergenerational online meetings, we speculate that many of the findings and design sensitivities might also apply to other contexts and situations. For instance, transferring this to work settings, we already know that objects and spaces are relevant (e.g., [13]), and we also agree that (human-non-human) interactions at work are situated (e.g., [54]). Related work already shows that it is promising to research how object-based remote collaboration might look like (e.g., [4, 16, 17]), and we assume that it is likely that temporal and spatial trajectories also play a role in how successful people collaborate. With our 
work, we have provided several perspectives and sensitivities that can future work can build upon in work, and further, contexts.

The aim of our study was to unpack the role of objects in their relation to grandparents and grandchildren. Given the qualitative nature of this study, we refrain from claiming that the findings are valid beyond our sample. However, we unveiled experiences, agencies, practices, and reservations, which we hope will help to question existing designs, and inspire further empirical studies and designerly approaches to intergenerational - hybrid - meetings.

\section{ACKNOWLEDGMENTS}

We are very grateful for the participants' willingness to share their experiences and stories with us. Additionally, we would like to thank Bieke Zaman, Marije Nouwen, Alina Krischkowsky and Martin Murer, as well as the anonymous reviewers for their support. We gratefully acknowledge the financial support by the Austrian Science Fund (FWF): I 3580-N33.

\section{REFERENCES}

[1] Madeleine Akrich and Bruno Latour. 1992. A summary of a convenient vocabulary for the semiotics of human and nonhuman assemblies. In Shaping Technology/Building Society Studies in Sociotecnical Change. The MIT Press, 259264

[2] Morgan G. Ames, Janet Go, Joseph 'Jofish' Kaye, and Mirjana Spasojevic. 2010. Making Love in the Network Closet: The Benefits and Work of Family Videochat In Proceedings of the 2010 ACM Conference on Computer Supported Cooperative Work (Savannah, Georgia, USA) (CSCW'10). Association for Computing Machin ery, New York, NY, USA, 145-154. https://doi.org/10.1145/1718918.1718946

[3] Grace Ataguba. 2018. Maintaining Long-Distance Relationships with Children: Exploring Autobiographical Designs and Life Logging. In Proceedings of the 17 th ACM Conference on Interaction Design and Children (Trondheim, Norway) (IDC '18). Association for Computing Machinery, New York, NY, USA, 693-698. https://doi.org/10.1145/3202185.3210801

[4] Huidong Bai, Prasanth Sasikumar, Jing Yang, and Mark Billinghurst. 2020. A User Study on Mixed Reality Remote Collaboration with Eye Gaze and Hand Gesture Sharing. In Proceedings of the 2020 CHI Conference on Human Factors in Computing Systems (Honolulu, HI, USA) (CHI '20). Association for Computing Machinery, New York, NY, USA, 1-13. https://doi.org/10.1145/3313831.3376550

[5] Rafael Ballagas, Joseph 'Jofish' Kaye, Morgan Ames, Janet Go, and Hayes Raffle. 2009. Family Communication: Phone Conversations with Children. In Proceedings of the 8th International Conference on Interaction Design and Children (Como, Italy) (IDC '09). Association for Computing Machinery, New York, NY, USA, 321-324. https://doi.org/10.1145/1551788.1551874

[6] Janne Mascha Beuthel, Philippe Bentegeac, Verena Fuchsberger, Bernhard Maurer, and Manfred Tscheligi. 2021 (to appear). Experiencing Distance: Wearable Engagements with Remote Relationships. In Proceedings of the International Conference on Tangible, Embedded and Embodied Interaction (TEI '21). ACM. https://doi.org/10.1145/3430524.3446071

[7] Margot Brereton, Alessandro Soro, Kate Vaisutis, and Paul Roe. 2015. The Messaging Kettle: Prototyping Connection over a Distance Between Adult Children and Older Parents. In Proceedings of the 33rd Annual ACM Conference on Human Factors in Computing Systems (Seoul, Republic of Korea) (CHI '15). ACM, New York, NY, USA, 713-716. https://doi.org/10.1145/2702123.2702462

[8] Saniye Tugba Bulu. 2012. Place presence, social presence, co-presence, and satisfaction in virtual worlds. Computers \& Education 58, 1 (2012), 154-161. https://doi.org/10.1016/j.compedu.2011.08.024

[9] Gillian Busch. 2018. How Families Use Video Communication Technologies During Intergenerational Skype Sessions. Springer Singapore, Singapore, 17-32. https: //doi.org/10.1007/978-981-10-6484-5_2

[10] Christopher J. Carpenter and Stephanie Tom Tong. 2017. Relational Distancing and Termination between Online Friends: An Application of the Investment Model. In Proceedings of the 2017 CHI Conference on Human Factors in Computing Systems (Denver, Colorado, USA) (CHI '17). Association for Computing Machinery, New York, NY, USA, 6925-6935. https://doi.org/10.1145/3025453.3026026

[11] Eva Cerezo and Ana Cristina Blasco. 2019. The Space Journey Game: An Intergenerational Pervasive Experience. In Extended Abstracts of the 2019 CHI Conference on Human Factors in Computing Systems (CHI EA '19). Association for Computing Machinery, New York, NY, USA, 1-6. https://doi.org/10.1145/3290607.3313055

[12] Liliana Costa and Ana Veloso. 2016. Being (Grand) Players: Review of Digital Games and their Potential to Enhance Intergenerational Interactions. Fournal of Intergenerational Relationships 14, 1 (2016), 43-59. https://doi.org/10.1080/ 15350770.2016.1138273 arXiv:https://doi.org/10.1080/15350770.2016.1138273

[13] Peter Dalsgaard, Kim Halskov, and Ditte Amund Basballe. 2014. Emergent Boundary Objects and Boundary Zones in Collaborative Design Research Projects. In Proceedings of the 2014 Conference on Designing Interactive Systems (Vancouver, BC, Canada) (DIS '14). Association for Computing Machinery, New York, NY, USA, 745-754. https://doi.org/10.1145/2598510.2600878

[14] Hillary Davis, Frank Vetere, Francis Peter, Martin Gibbs, and Steve Howard. 2008. "I Wish We Could Get Together": Exploring Intergenerational Play Across a Distance via a 'Magic Box'. Journal of Intergenerational Relationships 6, 2 (May 2008), 191-210. https://doi.org/10.1080/15350770801955321 Publisher: Routledge _eprint: https://doi.org/10.1080/15350770801955321.

[15] Linda M Drew and Merril Silverstein. 2007. Grandparents' psychological wellbeing after loss of contact with their grandchildren. Fournal of family psychology 21, 3 (2007), 372.

[16] Martin Feick, Terrance Mok, Anthony Tang, Lora Oehlberg, and Ehud Sharlin. 2018. Perspective on and Re-Orientation of Physical Proxies in Object-Focused Remote Collaboration. In Proceedings of the 2018 CHI Conference on Human Factors in Computing Systems (Montreal OC, Canada) (CHI '18). Association for Computing Machinery, New York, NY, USA, 1-13. https://doi.org/10.1145/ 3173574.3173855

[17] Martin Feick, Anthony Tang, and Scott Bateman. 2018. Mixed-Reality for ObjectFocused Remote Collaboration. In The 31st Annual ACM Symposium on User Interface Software and Technology Adjunct Proceedings (Berlin, Germany) (UIST '18 Adjunct). Association for Computing Machinery, New York, NY, USA, 63-65. https://doi.org/10.1145/3266037.3266102

[18] Sean Follmer, Hayes Raffle, Janet Go, Rafael Ballagas, and Hiroshi Ishii. 2010. Video Play: Playful Interactions in Video Conferencing for Long-Distance Families with Young Children. In Proceedings of the 9th International Conference on Interaction Design and Children (Barcelona, Spain) (IDC '10). Association for Computing Machinery, New York, NY, USA, 49-58. https://doi.org/10.1145/1810543. 1810550

[19] Azadeh Forghani and Carman Neustaedter. 2014. The Routines and Needs of Grandparents and Parents for Grandparent-Grandchild Conversations over Distance. In Proceedings of the SIGCHI Conference on Human Factors in Computing Systems (Toronto, Ontario, Canada) (CHI '14). Association for Computing Machinery, New York, NY, USA, 4177-4186. https://doi.org/10.1145/2556288.2557255

[20] Azadeh Forghani, Carman Neustaedter, and Thecla Schiphorst. 2013. Investigating the Communication Patterns of Distance-Separated Grandparents and Grandchildren. In CHI '13 Extended Abstracts on Human Factors in Computing Systems (Paris, France) (CHI EA '13). Association for Computing Machinery, New York, NY, USA, 67-72. https://doi.org/10.1145/2468356.2468370

[21] Azadeh Forghani, Carman Neustaedter, Manh C. Vu, Tejinder K. Judge, and Alissa N. Antle. 2018. G2G: The Design and Evaluation of a Shared Calendar and Messaging System for Grandparents and Grandchildren. In Proceedings of the 2018 CHI Conference on Human Factors in Computing Systems (CHI '18). Association for Computing Machinery, New York, NY, USA, 1-12. https://doi.org/10.1145/ 3173574.3173729

[22] Christopher Frauenberger. 2019. Entanglement HCI The Next Wave? ACM Trans. Comput.-Hum. Interact. 27, 1, Article 2 (Nov. 2019), 27 pages. https: //doi.org/10.1145/3364998

[23] Melissa Freeman. 2019. The analytic rewards of materializing the effects of actor-networks. Qualitative Research 19, 4 (2019), 455-470. https://doi.org/10. 1177/1468794118778977 arXiv:https://doi.org/10.1177/1468794118778977

[24] Verena Fuchsberger, Martin Murer, and Manfred Tscheligi. 2013. Materials, Materiality, and Media. In Proceedings of the SIGCHI Conference on Human Factors in Computing Systems (Paris, France) (CHI '13). ACM, New York, NY, USA, 28532862. https://doi.org/10.1145/2470654.2481395

[25] Verena Fuchsberger, Martin Murer, David Wilfinger, and Manfred Tscheligi. 2011. Attributes of Successful Intergenerational Online Activities. In Proceedings of the 8th International Conference on Advances in Computer Entertainment Technology (Lisbon, Portugal) (ACE '11). ACM, New York, NY, USA, Article 50, 8 pages. https://doi.org/10.1145/2071423.2071486

[26] Yumei Gan, Christian Greiffenhagen, and Stuart Reeves. 2020. Connecting Distributed Families: Camera Work for Three-Party Mobile Video Calls. In Proceedings of the 2020 CHI Conference on Human Factors in Computing Systems (Honolulu, HI, USA) (CHI '20). Association for Computing Machinery, New York, NY, USA, 1-12. https://doi.org/10.1145/3313831.3376704

[27] Richard Grusin. 2015. The nonhuman turn. U of Minnesota Press.

[28] Nik Rushdi Hassan. 2016. Editorial: A Brief History of the Material in Sociomateriality. SIGMIS Database 47, 4 (Dec. 2016), 10-22. https://doi.org/10.1145/ 3025099.3025101

[29] Alexis Hope, Ted Schwaba, and Anne Marie Piper. 2014. Understanding digital and material social communications for older adults. In Proceedings of the SIGCHI Conference on Human Factors in Computing Systems (CHI '14). Association for Computing Machinery, New York, NY, USA, 3903-3912. https://doi.org/10.1145/ 2556288.2557133 
[30] Seth E. Hunter, Pattie Maes, Anthony Tang, Kori M. Inkpen, and Susan M. Hessey. 2014. WaaZam! Supporting Creative Play at a Distance in Customized Video Environments. In Proceedings of the SIGCHI Conference on Human Factors in Computing Systems (Toronto, Ontario, Canada) (CHI '14). Association for Computing Machinery, New York, NY, USA, 1197-1206. https://doi.org/10.1145/2556288.2557382

[31] Jasmine Jones and Mark S. Ackerman. 2018. Co-constructing Family Memory: Understanding the Intergenerational Practices of Passing on Family Stories. In Proceedings of the 2018 CHI Conference on Human Factors in Computing Systems (CHI '18). Association for Computing Machinery, New York, NY, USA, 1-13. https://doi.org/10.1145/3173574.3173998

[32] Tejinder K. Judge, Carman Neustaedter, Steve Harrison, and Andrew Blose. 2011. Family Portals: Connecting Families through a Multifamily Media Space. In Proceedings of the SIGCHI Conference on Human Factors in Computing Systems (Vancouver, BC, Canada) (CHI '11). Association for Computing Machinery, New York, NY, USA, 1205-1214. https://doi.org/10.1145/1978942.1979122

[33] Jina Kim, Young-Woo Park, and Tek-Jin Nam. 2015. BreathingFrame: An Inflatable Frame for Remote Breath Signal Sharing. In Proceedings of the Ninth International Conference on Tangible, Embedded, and Embodied Interaction (Stanford, California USA) (TEI '15). Association for Computing Machinery, New York, NY, USA, 109-112. https://doi.org/10.1145/2677199.2680606

[34] Ji Eun Kim and Jim Anderson. 2008. Mother-child shared reading with print and digital texts:. Fournal of Early Childhood Literacy (Aug. 2008). https://doi. org/10.1177/1468798408091855 Publisher: SAGE PublicationsSage UK: London, England.

[35] Kari Kuutti and Liam J. Bannon. 2014. The Turn to Practice in HCI: Towards a Research Agenda. In Proceedings of the SIGCHI Conference on Human Factors in Computing Systems (Toronto, Ontario, Canada) (CHI '14). ACM, New York, NY, USA, 3543-3552. https://doi.org/10.1145/2556288.2557111

[36] Bruno Latour. 2007. Reassembling the Social. An Introduction to Actor-NetworkTheory. Oxford University Press.

[37] Hung-Chi Lee and Jane Yung-jen Hsu. 2016. PicMemory: Enriching Intergen erational Family Interaction and Memory Collection. In Proceedings of the 2016 CHI Conference Extended Abstracts on Human Factors in Computing Systems (CHI EA '16). Association for Computing Machinery, New York, NY, USA, 3715-3718. https://doi.org/10.1145/2851581.2890233

[38] Eva Lenz, Marc Hassenzahl, Wasili Adamow, Patrick Beedgen, Kirstin Kohler and Thies Schneider. 2016. Four Stories About Feeling Close Over A Distance. In Proceedings of the TEI '16: Tenth International Conference on Tangible, Embedded, and Embodied Interaction (Eindhoven, Netherlands) (TEI '16). Association for Computing Machinery, New York, NY, USA, 494-499. https://doi.org/10.1145/ 2839462.2856523

[39] A. Mol. 2010. Actor-Network Theory: sensitive terms and enduring tensions Kölner Zeitschrift für Soziologie und Sozialpsychologie. Sonderheft 50 (2010), 253269.

[40] Amelia K Moody, Laura M Justice, and Sonia Q Cabell. 2010. Electronic versus traditional storybooks: Relative influence on preschool children's engagement and communication. Fournal of Early Childhood Literacy 10, 3 (Sept. 2010), 294313. https://doi.org/10.1177/1468798410372162

[41] Preeti Mudliar and Nimmi Rangaswamy. 2015. Offline Strangers, Online Friends: Bridging Classroom Gender Segregation with WhatsApp. In Proceedings of the 33rd Annual ACM Conference on Human Factors in Computing Systems (Seoul, Republic of Korea) (CHI '15). Association for Computing Machinery, New York, NY, USA, 3799-3808. https://doi.org/10.1145/2702123.2702533

[42] Florian "Floyd" Mueller, Frank Vetere, Martin R. Gibbs, Jesper Kjeldskov, Sonja Pedell, and Steve Howard. 2005. Hug over a Distance. In CHI '05 Extended Abstracts on Human Factors in Computing Systems (Portland, OR, USA) (CHI EA '05). Association for Computing Machinery, New York, NY, USA, 1673-1676. https://doi.org/10.1145/1056808.1056994

[43] Stefanie Mueller, Martin Fritzsche, Jan Kossmann, Maximilian Schneider Jonathan Striebel, and Patrick Baudisch. 2015. Scotty: Relocating Physical Objects Across Distances Using Destructive Scanning, Encryption, and 3D Printing. In Proceedings of the Ninth International Conference on Tangible, Embedded, and Embodied Interaction (Stanford, California, USA) (TEI '15). ACM, New York, NY, USA, 233-240. https://doi.org/10.1145/2677199.2680547

[44] Carman Neustaedter and Saul Greenberg. 2012. Intimacy in Long-Distance Relationships over Video Chat. In Proceedings of the SIGCHI Conference on Human Factors in Computing Systems (Austin, Texas, USA) (CHI '12). Association for Computing Machinery, New York, NY, USA, 753-762. https://doi.org/10.1145/ 2207676.2207785

[45] nextnature.net. [n.d.]. Seven ways to connect with your loved ones (and others) https://nextnature.net/2020/05/seven-ways-to-connect-with-your-loved-ones

[46] Thammathip Piumsomboon, Gun A. Lee, Jonathon D. Hart, Barrett Ens, Robert W. Lindeman, Bruce H. Thomas, and Mark Billinghurst. 2018. Mini-Me: An Adaptive Avatar for Mixed Reality Remote Collaboration. In Proceedings of the 2018 CHI Conference on Human Factors in Computing Systems (Montreal QC, Canada) (CHI '18). Association for Computing Machinery, New York, NY, USA, 1-13. https://doi.org/10.1145/3173574.3173620
[47] Ernesto Priego and Peter Wilkins. 2020. Comics as Covid-19 Response: Visualizing the Experience of Videoconferencing with Aging Relatives. Interactions 27, 4 (July 2020), 60-61. https://doi.org/10.1145/3404207

[48] Hayes Raffle, Glenda Revelle, Koichi Mori, Rafael Ballagas, Kyle Buza, Hiroshi Horii, Joseph Kaye, Kristin Cook, Natalie Freed, Janet Go, and Mirjana Spasojevic. 2011. Hello, is Grandma There? Let's Read! StoryVisit: Family Video Chat and Connected e-Books. In Proceedings of the SIGCHI Conference on Human Factors in Computing Systems (Vancouver, BC, Canada) (CHI '11). ACM, New York, NY, USA, 1195-1204. https://doi.org/10.1145/1978942.1979121

[49] Crystal N. Reisig and Bronwyn S. Fees. 2007. Older Adults' Perceptions of WellBeing after Intergenerational Experiences with Youth. Fournal of Intergenerational Relationships 4, 4 (March 2007), 6-22. https://doi.org/10.1300/J194v04n04_02 Publisher: Routledge_eprint: https://doi.org/10.1300/J194v04n04_02.

[50] Glenda Revelle and Jennifer Bowman. 2017. Parent-Child Dialogue with eBooks. In Proceedings of the 2017 Conference on Interaction Design and Children (IDC '17). Association for Computing Machinery, Stanford, California, USA, 346-351. https://doi.org/10.1145/3078072.3079753

[51] I. Rodríguez, M. Oteo, S. Gleisner, and V. Herskovic. 2015. SINCOM: Communicating grandparents and grandchildren living at a distance. In 2015 IEEE 19th International Conference on Computer Supported Cooperative Work in Design (CSCWD). 153-158. https://doi.org/10.1109/CSCWD.2015.7230950

[52] Edwin Sayes. 2014. Actor-Network Theory and methodology: Just what does it mean to say that nonhumans have agency? Social Studies of Science 44, 1 (2014), 134-149. http://www.jstor.org/stable/43284223

[53] Samarth Singhal, Carman Neustaedter, Yee Loong Ooi, Alissa N. Antle, and Brendan Matkin. 2017. Flex-N-Feel: The Design and Evaluation of Emotive Gloves for Couples to Support Touch Over Distance. In Proceedings of the 2017 ACM Conference on Computer Supported Cooperative Work and Social Computing (Portland, Oregon, USA) (CSCW'17). Association for Computing Machinery, New York, NY, USA, 98-110. https://doi.org/10.1145/2998181.2998247

[54] Lucille Alice Suchman. 1987. Plans and situated actions: the problem of humanmachine communication. Cambridge university press.

[55] Anna Taylor and Amanda Gummer. 2018. Connected Grandparents: Are Smart Toys the Future for Intergenerational Play?. In 8th International Toy Research Association World Conference, Gilles Brougère and Mark Allen (Eds.). International Toy Research Association (ITRA), Paris, France. https://hal-univ-paris13.archivesouvertes.fr/hal-02081469

[56] Javier Tibau, Michael Stewart, Steve Harrison, and Deborah Tatar. 2019. FamilySong: A Design for Managing Synchronous Intergenerational Remote Music Sharing. In Companion Publication of the 2019 on Designing Interactive Systems Conference 2019 Companion (DIS '19 Companion). Association for Computing Machinery, San Diego, CA, USA, 61-64. https://doi.org/10.1145/3301019.3325159

[57] Theodora Vardouli. 2015. Making use: Attitudes to human artifact engagements. Design Studies 41, Part A (2015), 137 - 161. https://doi.org/10.1016/j.destud.2015. 08.002 Special Issue: Computational Making.

[58] Frank Vetere, Mark Nolan, and Raihaan Abdool Raman. 2006. Distributed Hideand-Seek. In Proceedings of the 18th Australia Conference on Computer-Human Interaction: Design: Activities, Artefacts and Environments (Sydney, Australia) (OZCHI '06). Association for Computing Machinery, New York, NY, USA, 325328. https://doi.org/10.1145/1228175.1228235

[59] Yvonne Vezzoli, Sara Kalantari, Natalia Kucirkova, and Asimina Vasalou. 2020 Exploring the Design Space for Parent-Child Reading. In Proceedings of the 2020 CHI Conference on Human Factors in Computing Systems (CHI '20). Association for Computing Machinery, New York, NY, USA, 1-12. https://doi.org/10.1145/ 3313831.3376696

[60] Torben Wallbaum, Andrii Matviienko, Swamy Ananthanarayan, Thomas Olsson, Wilko Heuten, and Susanne C.J. Boll. 2018. Supporting Communication between Grandparents and Grandchildren through Tangible Storytelling Systems. In Proceedings of the 2018 CHI Conference on Human Factors in Computing Systems (Montreal QC, Canada) (CHI '18). Association for Computing Machinery, New York, NY, USA, 1-12. https://doi.org/10.1145/3173574.3174124

[61] Greg Walsh and Elizabeth Foss. 2015. A case for intergenerational distributed co-design: the online kidsteam example. In Proceedings of the 14th International Conference on Interaction Design and Children (IDC '15). Association for Computing Machinery, Boston, Massachusetts, 99-108. https://doi.org/10.1145/2771839. 2771850

[62] Pan Wang, Gerald Choon-Huat Koh, Christian Gilles Boucharenc, and ChingChiuan Yen. 2017. Designing Two-player Competitive Games for the Rehabilitation of Upper-Limb Motor Function after Stroke. In Proceedings of the 2017 CHI Conference Extended Abstracts on Human Factors in Computing Systems (CHI EA '17). Association for Computing Machinery, New York, NY, USA, 2201-2209. https://doi.org/10.1145/3027063.3053069

[63] Jun Wei, Xuan Wang, Roshan Lalintha Peiris, Yongsoon Choi, Xavier Roman Martinez, Remi Tache, Jeffrey Tzu Kwan Valino Koh, Veronica Halupka, and Adrian David Cheok. 2011. CoDine: An Interactive Multi-Sensory System for Remote Dining. In Proceedings of the 13th International Conference on Ubiquitous Computing (Beijing, China) (UbiComp '11). Association for Computing Machinery, New York, NY, USA, 21-30. https://doi.org/10.1145/2030112.2030116 
[64] Tiffanie Wen. [n.d.]. How coronavirus has transformed the way we communicate. https://www.bbc.com/worklife/article/20200408-coronavirus-howlockdown-helps-those-who-fear-the-phone

[65] Naomi Yamashita, Hideaki Kuzuoka, Keiji Hirata, Shigemi Aoyagi, and Yoshinari Shirai. 2011. Supporting Fluid Tabletop Collaboration across Distances. In Proceedings of the SIGCHI Conference on Human Factors in Computing Systems (Vancouver, BC, Canada) (CHI '11). Association for Computing Machinery, New York, NY, USA, 2827-2836. https://doi.org/10.1145/1978942.1979362

[66] Lillian Yang and Carman Neustaedter. 2020. An Autobiographical Design Study of a Long Distance Relationship: When Telepresence Robots Meet Smart Home Tools. In Proceedings of the 2020 ACM Designing Interactive Systems Conference (Eindhoven, Netherlands) (DIS '20). Association for Computing Machinery, New
York, NY, USA, 129-140. https://doi.org/10.1145/3357236.3395467

[67] Lining Yao, Sayamindu Dasgupta, Nadia Cheng, Jason Spingarn-Koff, Ostap Rudakevych, and Hiroshi Ishii. 2011. Rope Revolution: Tangible and Gestural Rope Interface for Collaborative Play. In Proceedings of the 8th International Conference on Advances in Computer Entertainment Technology (Lisbon, Portugal) (ACE '11). Association for Computing Machinery, New York, NY, USA, Article 11, 8 pages. https://doi.org/10.1145/2071423.2071437

[68] Ye Yuan and Svetlana Yarosh. 2019. Beyond Tutoring: Opportunities for Intergenerational Mentorship at a Community Level. In Proceedings of the 2019 CHI Conference on Human Factors in Computing Systems (CHI '19). Association for Computing Machinery, New York, NY, USA, 1-14. https://doi.org/10.1145/3290605.3300679 